\title{
Una propuesta epistemológica para la sistematización de experiencias generada desde la reflexión sobre la práctica'
}

Carmenza Sánchez Rodríguez ${ }^{2}$

Recibido: 15-08-2016

Aceptado: 03-11-2016

\section{RESUMEN}

Este documento es fruto de un proceso de reflexión sobre la práctica en el marco del proceso de sistematización de la experiencia "Configuración de las nociones formación, didáctica, pedagogía y práctica pedagógica: Sistematización de la experiencia en la Especialización en Pedagogía $(2010-2015)^{\prime \prime}$, que plantea los referentes ontológicos y epistemológicos del proyecto. El documento hace explícitas las comprensiones del grupo de investigadores sobre las concepciones de la investigación, la naturaleza de la realidad y del conocimiento, las visiones de mundo, los métodos, el rol de los sujetos, los alcances, los lugares y las fuentes de producción del conocimiento, que constituyen los referentes epistemológicos desde los cuales se asume la sistematización de experiencias. En esta reflexión, especialmente en lo relacionado con las formas de producción de conocimiento, toma especial relevancia la reflexión sobre la práctica, como forma de producción de conocimiento que ha marcado los desarrollos epistemológicos y pedagógicos en la Especialización en los últimos años.

Palabras clave: Epistemología, producción de conocimiento, sistematización de experiencias, reflexión sobre la práctica.

1. Artículo corto, avance de investigación del proyecto Configuración de las nociones formación, didáctica, pedagogía y práctica pedagógica: Sistematización de la experiencia en la Especialización en Pedagogía (2010 - 2015).

2. Filósofa, Magister en Educación, miembro del Grupo Filosofía, Sociedad y Educación. Docente del Departamento de Posgrado de la Universidad Pedagógica Nacional de Colombia. Proyecto de investigación financiado por el Centro de Investigaciones de la Universidad Pedagógica Nacional.

Correo: csanchez@pedagogica.edu.co 


\title{
Proposal for a systematizing the experience epistemological generated from practice on reflection
}

\begin{abstract}
This text is product of the project of research settings of them notions training, didactic, pedagogy and practice pedagogical: systematization of the experience in the specialization in pedagogy (2010-2015), especially reflects on the concept of practice, their relating ontological and epistemological. The document makes explicit understandings of the group of researchers on the conceptions of the research, the nature of reality and knowledge, visions of the world, the methods, the role of subjects, reaches, places and sources of knowledge production, which constitute the epistemological references from which it is assumed the systematization of experiences. In this direction, taking particularly relevant reflection on practice, as a form of knowledge production, which has marked the epistemological and pedagogical developments in the specialization in recent years.
\end{abstract}

Keywords: Epistemology, knowledge production, systematization of experiences, reflection on practice.

\section{Proposta epistemológica para a sistematização de experiências gerada desde a reflexão sobre a prática}

\section{RESUMO}

Este documento é o resultado de um processo de reflexão sobre a prática no marco do processo da sistematização da experiência, "configuração das noções de formação, didática, pedagogia e pratica pedagógica: sistematização da experiência na Especialização em Pedagogia (20101015)", que expressa os referentes ontológicos e epistemológicos do projeto. O documento faz explicitas as idéias do grupo de pesquisadores 
sobre as compressões de pesquisa, a natureza da realidade y do conhecimento, as visões do mundo, os métodos, o papel dos sujeitos, escopo, os lugares e as fontes da produção do conhecimento, que são a referencia epistemológica a partir da qual a sistematização de experiências é assumida. Nesta reflexão, especialmente em relação ás formas de produção de conhecimento que tem marcado a evolução epistemológica e pedagógica na Especialização nos últimos anos.

Palavras-chave: Epistemologia, produção de conhecimento, sistematização de experiências, reflexão sobre a prática.

\section{Introducción}

Este documento es producto de las discusiones del grupo de investigadores de la Especialización en Pedagogía de la Universidad Pedagógica Nacional: Guillermo Fonseca, Gloria Orjuela Sánchez, José Gómez Franco, Luz Betty Ruiz Pulido, Pablo Ortiz Orjuela y la autora, en el marco del Proyecto CIUP, denominado "Configuración de las nociones formación, didáctica, pedagogía y práctica pedagógica: Sistematización de la experiencia en la Especialización en Pedagogía (2010 - 2015)", y surge del diálogo de saberes realizado, en principio, en dos instancias, la primera con los textos ${ }^{3}$ de Alfredo Ghiso, Marco Raúl Mejía, Lola Cendales, Guillermo Torres, Oscar Jara, María Mercedes Barnechea, que aparecen como referentes provocadores para la discusión de algunos elementos considerados esenciales en la construcción del horizonte de sentido del proyecto $y$, la segunda, en la reflexión generada por el grupo de investigadores sobre los referentes epistemológicos de la sistematización de experiencias que convocó un diálogo en torno a los saberes construidos sobre la práctica, en la reflexión sobre las apuestas formativas en investigación del programa Especialización en Pedagogía.

Esta apuesta epistemológica obliga a hacer claridades sobre el uso de las citas en el documento, ya que el lector no encontrará ninguna cita de autoridad que se use para dar validez a los argumentos del escrito o para mostrar los puntos de coincidencia con los postulados de un autor. Sin embargo, esta apuesta epistemológica reconoce que el saber

3. Estos textos se referencian en la bibliografía pero sólo como referentes para la reflexión ya que no se toman citas de los mismos. 
construido, fruto de la reflexión sobre la práctica (entendida como discurso y acción), es producto de un saber heredado culturalmente y de un saber que se reconfigura en la acción - reflexión. Por ello, aunque aquí no aparezcan citas ${ }^{4}$, ni parafraseos, se entiende que en esta construcción aparecen leídos, reconfigurados, deconstruidos, reconcepctualizados y recontextualizados los saberes heredados de los diferentes campos de formación de los investigadores y de los saberes movilizados en sus prácticas profesionales, así como los saberes acumulados por quienes de una $u$ otra manera han aportado a las discusiones sobre la naturaleza del saber, la ciencia, las visiones de mundo desde las cuales se produce el conocimiento y los lugares de producción de los sujetos. En este sentido las ideas de Popper, Khun, la Escuela de Frankfurt y la teoría crítica, Vasilachis y Foucault, entre otros, así como las construcciones del Observatorio Nacional de Políticas en Evaluación de la Universidad Pedagógica Nacional, están presentes en esta discusión y, aunque no de manera absolutamente coherente, se han articulado en esta construcción sobre los referentes epistemológicos de la sistematización de experiencias. Este documento no se presenta como una propuesta epistemológica novedosa sino como una comprensión de las discusiones dadas en torno a este asunto, tanto desde la sistematización de experiencias como desde la discusión epistemológica tradicional, elaborada por el grupo de docentes investigadores.

De este modo, el documento hace explícitas las comprensiones del grupo de investigadores sobre las concepciones de la investigación, la naturaleza de la realidad y del conocimiento, las visiones de mundo, los métodos, el rol de los sujetos, los alcances, los lugares y las fuentes de producción del conocimiento, que constituyen los referentes epistemológicos desde los cuales se asume la investigación y la sistematización de experiencias.

\section{La producción de conocimiento como producción política}

Este proyecto comprende la producción de conocimiento como una "producción política5" que no solamente está relacionada con el empoderamiento de los sujetos para develar las ideologías presentes 
o para comprender su realidad y transformarla, sino que asume que el conocimiento en símismo es una producción política que moviliza sentidos y significados sobre el sujeto cognoscente, sobre las epistemologías desde donde se produce, los métodos de producción, la naturaleza misma del conocimiento y, en últimas, sobre la naturaleza de la realidad.

Lo político también tiene que ver con las formas de relación que se crean desde esa producción de conocimiento, lo que se organiza y cómo se organiza, sin asumirlo como una intencionalidad previa sino como algo que va cambiando de acuerdo a las formas de organización y relación. Desde esta perspectiva de lo político, el conocimiento no sólo produce saber sino que crea formas de comprender y relacionarse en el mundo que no necesariamente responde a una intencionalidad previa de los científicos o las comunidades científicas.

En este sentido, la visión eurocéntrica del conocimiento, fuertemente influida por el positivismo, no sólo produjo una forma de producción de conocimiento sino que la instauró como un discurso político sobre las formas válidas de su producción y sobre la naturaleza universalmente válida del mismo. Esta visión sobre el conocimiento y la ciencia hizo que los sujetos se comportarán frente al mundo como algo dado, no atravesado por la subjetividad de quien conoce y frente al conocimiento como algo acabado y producido en una instancia superior, diferente al mundo del sujeto que lo produce. Así, se fueron instituyendo nuevas relaciones y formas de organización frente al mundo y al conocimiento. El conocimiento se asumió como una producción objetiva y neutral que iba más allá de cualquier contingencia temporal o histórica y las formas de producción de saber se redujeron a un método, obviando la discusión epistemológica de su producción.

Esta visión de conocimiento produjo también las conocidas dicotomías entre conocimiento - saber, ciencia - no ciencia, sujeto - objeto, subjetividad - objetividad, teoría - práctica, etc., negando además que la producción de conocimiento está social e históricamente condicionada y vinculada a una visión de mundo. Así, estas visiones sobre el conocimiento y el mundo se instauraron no solamente como producción científica sino como producción política que se convirtió en discurso hegemónico sobre la naturaleza del conocimiento y la realidad.

Cuando las ciencias sociales empiezan a hacer frente a esta visión hegemónica del conocimiento a partir de todas las críticas hechas 
al positivismo, se establece una concepción de conocimiento de segundo orden, el saber, que podía ser producto del ejercicio riguroso de los científicos sociales, pero que no podía ostentar el carácter de conocimiento. Las ciencias sociales inician un proceso de reivindicación de las características de los fenómenos sociales que impiden que ellas produzcan conocimiento universalmente válido y explicitan las razones por las cuales renuncia a esta pretensión.

Este proyecto asume como ficticia la distinción conocimiento - saber (y otras dicotomías impulsadas desde el positivismo como teoría práctica, sujeto - objeto, entre otras), reconociendo el carácter situado y provisionalmente válido (Popper, 1986) del conocimiento, que impiden que se produzca con pretensiones de objetividad o neutralidad, de explicación de fenómenos o de verdades sobre el mundo. Se entiende que ningún conocimiento producido puede ser considerado universalmente válido y necesario o como una explicación de una realidad dada y por esa razón se usará indistintamente "conocimiento" o "saber" como sinónimos, refiriéndonos a la producción científica, en cualquier campo (ciencias sociales o naturales), que tiene como características su carácter situado y provisional (aunque sistemático y riguroso).

\section{El rescate de una visión integradora del conocimiento: acción - pensamiento - emoción}

La visión dicotómica del conocimiento, en la concepción eurocéntrica, instaló la idea de que el conocimiento se produce por fuera de las emociones y las visiones de mundo de los sujetos y que es el "mundo dado" el que informa a los sujetos sus leyes de organización y funcionamiento. Se escindió el sujeto y se establecieron diferentes esferas: lo cognitivo, lo afectivo, lo político, lo ético, etc., como campos separados que no tenían incidencia en la producción de conocimiento. Las críticas al positivismo y a la visión eurocéntrica de la ciencia han dejado claro que en la producción de conocimiento inciden todos estos factores y que es imposible sostener una visión del conocimiento como práctica objetiva que se realiza por fuera de las emociones, visiones de mundo y posicionamientos epistemológicos, políticos y axiológicos de los sujetos "conocidos" (Vasilachis, 2006).

Este proyecto piensa la producción de conocimiento como una actividad política, emotiva, volitiva, ética, epistemológica y ontológica, es decir, 
humana. El sujeto cognoscente se comprende como totalidad en su proceso de construcción de la realidad o en la vivencia de una experiencia. El conocimiento supone y convoca las concepciones políticas, éticas, ontológicas y epistemológicas de los sujetos, por ello, no solamente está cargado de intereses de todo tipo sino que es producido desde ellos. La actividad científica lleva implícitas de manera inescindible las emociones, representaciones, las visiones de mundo y las culturas de los sujetos, por lo que no es posible hallar un sujeto cognoscente desprendido de su subjetividad para la producción de conocimiento.

\section{La actividad científica como actividad situada}

El reconocer al conocimiento como social e históricamente producido hace que no se pueda desligar de los contextos en los que los sujetos lo producen. En este sentido, no es preciso justificar las especificidades de una cultura como la latinoamericana para legitimar la producción de conocimiento y su naturaleza científica, simplemente se asume que el saber, siendo social e históricamente determinado, se produce en contextos diferentes y que su producción está atada a las contingencias histórico sociales de una cultura específica. Esta no es una característica propia de la actividad científica en Latinoamérica sino que es una condición inherente a la producción de conocimiento en cualquier lugar del mundo. El conocimiento producido está atado a unas condiciones histórico sociales que hacen que los sujetos se representen la realidad de una manera y todas sus construcciones se soportan sobre esa representación, aunque se produzca con pretensiones hegemónicas y se ponga a circular con la idea de convertirse en "ciencia normal" (Kuhn, 1970).

Desde esta perspectiva, se asume que el conocimiento producido es de naturaleza científica porque es producto de una reflexión sistemática y rigurosa sobre un fenómeno y entiende que, su carácter contextual, la implicación de los sujetos y sus subjetividades, son sus características esenciales. Renuncia a las pretensiones de universalidad y objetividad, no sólo porque el fenómeno social es imposible de atrapar asépticamente o aprehender en un momento histórico para conservarlo como una fotografía de una realidad dada, sino porque todo conocimiento producido responde a una concepción de la realidad y a unas lógicas histórico sociales concretas que lo condicionan. 


\section{La apuesta por el posicionamiento político del sujeto cognoscente}

Para esta investigación el sujeto cognoscente no es un sujeto abstracto, neutral o ahistórico, sino que se entiende como concreto, posicionado políticamente y contextualizado, es un sujeto "conocido" (Vasilachis, 2006), que no se distingue de un sujeto "cognoscente".

En este sentido, el posicionamiento político del sujeto se evidencia en dos aspectos:

1. Posicionamiento frente a los discursos hegemónicos. La comprensión de la producción de conocimiento como producción política también tiene dos formas de expresión en el posicionamiento de los sujetos: una, frente a los discursos hegemónicos sobre la producción y la naturaleza del mismo, que entiende que la producción de conocimiento se realiza en cualquier campo de saber y por cualquier grupo de científicos que asumen una reflexión sistemática y rigurosa sobre un "objeto" o fenómeno social y, otra, en el reconocimiento por parte de los sujetos de que la construcción de conocimiento produce sentidos y significados que se ponen a circular a partir de una determinada visión de mundo.

2. El reconocimiento de intencionalidades en la producción de conocimiento. Este proyecto surge de un grupo de maestros que se vincula a la investigación con unas intencionalidades explícitas y unos propósitos comunes. Sin embargo, esta intencionalidad se entiende como "intención puesta en tensión", no como intencionalidad final sino como intención que se moviliza a través de tensiones producidas por discursos y visiones de mundo que coexisten $y$, aunque no de manera coherente, se vinculan. Se trabaja, en principio desde cuatro ideas:

a. El reconocimiento de un conocimiento pedagógico construido, es decir, el rescate del conocimiento de su práctica y la práctica como construcción de conocimiento.

b. La intencionalidad de un posicionamiento político de los investigadores entendida como el reconocimiento de la naturaleza misma del comprender y construir explicaciones.

c. El reconocimiento de los maestros como productores de conocimiento. 
d. La conciencia en el sujeto de su capacidad para transformar la realidad en la producción de conocimiento.

\section{La realidad como construcción social - dinámica}

La construcción de conocimiento está estrechamente vinculada a la concepción de la realidad. Esta concepción determina cómo me acerco a conocerla y por ello es importante responder a la pregunta por la misma: ¿La realidad es un mundo afuera, construido por fuera del sujeto o un mundo construido por él?

Este proyecto comprende que la realidad como social e históricamente construida supone, como característica esencial, su naturaleza dinámica, dialéctica, cambiante y en permanente construcción a partir de los procesos de interacción y construcción, abandonando la idea de una realidad dada y de una función explicativa de ella, para el conocimiento. Desde esta concepción, el proceso de construcción de conocimiento es un proceso de transformación permanente de esa realidad y de los sujetos que la construyen. En la interacción y en la producción de conocimiento se generan nuevos sentidos y significados que se ponen a circular, creando nuevas formas de relación y organización que producen una dinámica permanente de reflexión - acción- transformación, de la realidad y de los sujetos.

En esta perspectiva hay una doble interacción, el conocimiento no se construye por fuera de la realidad construida, es producto de esta construcción, y la realidad se reconstruye cuando se produce conocimiento sobre ella. Realidad, conocimiento y sujeto son producto de la interacción y se transforman en la producción de conocimiento.

\section{La naturaleza de la práctica como productora de conocimiento 6}

Esta es una propuesta epistemológica que se plantea, con el propósito de contribuir a la producción de saber socioculturalmente pertinente, por

6. Este apartado recoge la construcción realizada en torno al tema por los docentes de la Especialización en Pedagogía durante el primer y segundo semestre de 2015, recopilada por los profesores Gloria Orjuela Sánchez y Guillermo Fonseca y presentada en el Tercer Coloquio de Pedagogía realizado el 13 de noviembre de 2015 en Bogotá. 
vías de la reflexión sensible, acerca de lo realizado por sujetos concretos en espacio - temporalidades concretas. De ahí que la práctica es el objeto de análisis y reflexión que remite a su reconstrucción como hecho y potencialidad, por lo cual este concepto permite un doble movimiento complementario: a) objetivar reflexivamente lo inmediato de su materialización, y b) abrir paso a un proceso de subjetivación por parte del maestro como investigador. A través de esta propuesta invitamos a una objetivación del sí mismo en contexto, razón por la que la práctica no lo es solo porque proviene o se alimenta de unos saberes, sino también porque permite aprender desde lo concreto de nuestro actuar con base en la articulación de posibilidades de conocer lo conocido (reconocer críticamente) para producir transformaciones.

Proponer la reflexión sobre la práctica como método para la producción de conocimiento exige la respuesta a asuntos como el tipo y lugar del saber a construir, la relación entre el sujeto que indaga y ese saber, la intencionalidad o finalidad de las indagaciones y finalmente una respuesta por lo metodológico, es decir, el paso a paso, el camino a recorrer.

La reflexión sobre la práctica involucra dos vocablos que requieren ser precisados comprensivamente a fin de establecer sus aportes, alcances y limitaciones. El primero, la práctica, es una capacidad humana corporeizada, fácticamente ejercida -o realizable- en diversos ámbitos de interacción humana que, a su vez, se encuentran localizados en coordenadas histórico-culturales y sociopolíticas de diversa amplitud. Es en el seno de esta conexión de lo humano donde la práctica se inscribe, adquiere sentido y materialidad.

La noción de práctica no sólo se limita a la acción sino que está determinada por discursos y éstos son producidos desde visiones de mundo. Así se entiende la práctica como discurso y como acción, y la forma como el discurso y la acción se vinculan en ella. La práctica no es un hecho sino una construcción en la que se vinculan necesidades, interés, discursos, acciones y relaciones.

De otro lado, la práctica está ligada históricamente al oficio. Apropiar la palabra práctica en el contexto de la producción de una comunidad científica implica la consideración de la actividad como un oficio, como el conjunto de acciones que tienen lugar en espacios pensados para la construcción de conocimiento, que remite directamente a los sujetos 
que hacen posible que estas acciones constituyan una práctica y ésta a su vez un saber.

La denominada concreción en la práctica sugiere un "devenir" que fluctúa permanentemente entre lo probable, lo realizable; lo alcanzable y lo alcanzado; que sin lugar a dudas está presente permanentemente y exige revaluación, reconsideración y por ende nuevas determinaciones; orientadas por concepciones que trazan rutas, apuestas menos permeables a la estática y necesariamente más cercanas a la dinámica, entendida la primera como suspensión y la segunda como disposición al cambio.

La práctica como recorrido y lugar de comprensión, va más allá de las acciones y actividades propias de la cotidianidad para situarse en el campo profesional y disciplinar. Como lugar, se define en el espacio tiempo histórico, presencia y tensión de múltiples miradas, intensiones, provocaciones; escenario de objetos y sujetos, de sus relaciones, rupturas y contradicciones. En él se entretejen prácticas sociales, sentires, concepciones, ideas, juicios, nociones, expectativas, maneras de ver, de pensar, de actuar, en últimas, un escenario de reproduccióntransformación-adaptación social y cultural atravesado por múltiples discursos que lo reclaman, lo regulan, lo legitiman, lo apropian.

Como recorrido de comprensión, la práctica sitúa y da sentido a la experiencia profesional, esto es: 1) moviliza conocimientos (los produce y los apropia), para dar cuenta de sus lógicas y modos de operar en un contexto particular; 2) compromete estos conocimientos con la realidad, a fin de entender las variaciones, las contradicciones, las rupturas; 3) pone en diálogo conocimientos y saberes derivados de la experiencia con la teoría existente a fin de enriquecerla; 4) interpela desde el saber acumulado para interpretar la complejidad del escenario en el que se disponen los discursos que la atraviesan; y 5) se interroga a sí misma, como lugar particular de enunciación, para nutrir desde la reflexión nuevas miradas, para movilizar otras formas de apropiación, para crear y recrear, para significar y resignificar la experiencia creativa de construcción de conocimiento.

El saber requiere de su concreción en lo práctico, una objetivación que no se da sólo desde el hacer sino que cobra mayor sentido en el ejercicio reflexivo de lo que se dice, de lo que se sabe y de lo que se hace. De otra parte, la práctica por sí misma construye un saber que bien puede y 
requiere entrar en relación con el saber construido o dado, unas veces para confrontarlo, corroborarlo o replantearlo. Pero además para transformarlo, pues desde el saber que se construye en la práctica se logran aportes a solución de asuntos concretos de un contexto que tal vez no hayan sido pensados ni aportados por el saber construido o dado. La práctica tiene que ver con otros. Es una acción junto con otros. Es dialógica. El encuentro con un rostro interpelante. Se aborda en relación con otros, con la interpelación de otros, en la confrontación, no es mera reflexión abstracta y teórica, es una acción con otros, constitutiva de sujetos.

\section{La reflexión como perspectiva metodológica}

La reflexión como perspectiva metodológica sitúa la práctica como objeto de reflexión para hacerla comprensible y en ella se sitúan cinco asuntos sustanciales: el primero referido a su naturaleza, el segundo respecto a los objetos de conocimiento sobre los cuales recae la reflexión, el tercero sobre sus propiedades, el cuarto sobre los niveles y el quinto sobre la finalidades.

La naturaleza. La reflexión se configura en el acto del pensar de forma sistemática, que se vincula con el deseo de preguntarse, es una acción que convoca a la necesidad de su propia constitución como sujeto. La reflexión en este sentido cumple dos funciones interdependientes la producción de saber y la transformación del sujeto que reflexiona. Así, en esta idea del pensar como un proceso es posible reconocer los siguientes rasgos o principios:

- El principio de la existencia de una práctica vivida.

- El principio de suspensión, de extrañamiento, objetivación sobre esta práctica.

- El principio de conversación o dialogicidad.

- El principio de circularidad.

El desarrollo de los anteriores principios es posible en una demarcación desde la epistemología de la práctica, en la que se reconoce que la práctica en sí misma contiene un saber, que se valida en razón de la propia introspección del sujeto en un dialogo con el otro, en donde los principios de validez no se ubican en las generalizaciones sino en los procesos de 
transformación local, en donde la teoría no es el fin, sino una fuente con la cual se dialoga.

\section{Características:}

La propiedad de la incertidumbre: esta propiedad permite el reconocimiento del propio sujeto en relación con la configuración particular de la práctica, es decir, es el sujeto maestro quien construye una práctica singular, particular, idiosincrática, así la distinción sujetoobjeto, desaparece, en razón que él hace parte del objeto mismo, él es práctica.

La propiedad de lo incierto: Esta propiedad se asocia con la cualidad relativa de la indeterminación, en donde la práctica en si misma se produce a través de las propiedades emergentes que se suceden en la interacción de sus componentes.

La propiedad de la complejidad: La práctica en razón de su finalidad formativa vincula diferentes factores de orden personal, institucional, político, social, cultural, factores que se entrecruzan configurando una complejidad en su arquitectura, en la cual es la complejidad y no la simplificación lo que le permite constituirse en sí misma.

La propiedad de síntesis temporal y espacial: Esta propiedad reconoce que la práctica se constituye en una síntesis temporal y espacial, que está siendo a través del pasado en el presente, pero ese presente se proyecta en un futuro. Es decir el maestro en su práctica recurre al pasado para vivir el presente, pero ésta a su vez es ya pasado, y simultáneamente una proyección de futuro.

\section{Los niveles de reflexión}

Esta reflexión tiene varios niveles: en principio un nivel del sujeto consigo mismo, pero este solo no es suficiente porque se necesita de un conversar con el otro. El primer lugar tiene que ver con un querer preguntarse, que a su vez involucra a un tercero que me intenciona, tiene que ver con un elemento emotivo, tiene que ver con una condición de deseo de ese sujeto consigo mismo. Tiene que ver con el interés, el deseo que le motiva a hacerse esa pregunta por el reconocimiento del lugar en los diferentes 
escenarios; y por afirmarse que es un lugar en el que se construye un saber.

Nuestra incertidumbre o nuestras comprensiones o nuestras maneras de entender, son las tensiones que hemos planteado, que hemos decidido, para llevar a cabo el ejercicio de preguntarse, la pregunta vuelta sobre si (primer nivel) que se reafirma en un segundo nivel (la reflexión con el otro o junto con los otros). Estos dos niveles se convierten en un momento de suspensión para pensar lo que hacemos.

El objeto sobre el cual se reflexiona es sobre, en y para la práctica, que no son sitios excluyentes sino que son lugares de ubicación para el tipo de análisis que se quiere adelantar. Lugar en el que se pueden situar los elementos de tiempo y espacio. Es decir, un lugar desde el cual se ubica el sujeto en la práctica, que requiere del deseo para poder llevarlo a la reflexión y por ende a la producción de un saber.

Es un continuo en el que no hay un principio ni un fin. Está en movimiento constante. Es rizomático, se asemeja a una red que tiene la capacidad de adoptar la forma de lo que contiene, una forma que es construida.

Estas comprensiones sobre el conocimiento, la realidad y el lugar de los sujetos en su producción, realizadas por el equipo de maestros, orientarán el proceso de sistematización, entendida como una modalidad investigativa que construye conocimiento desde las experiencias vividas y luego reflexionadas y sistematizadas por sus actores. 


\section{Referencias bibliográficas}

Barnechea, M., González, E. y Morgan, M. (1994). La sistematización como producción de conocimientos. Revista La Piragua №. 9. Consejo de Educación de Adultos de América Latina (CEAAL), Santiago. Disponible en: http://aprendeenlinea.udea.edu.co/ $\mathrm{Ims} / \mathrm{moodle} / \mathrm{mod} / \mathrm{resource} / \mathrm{view}$.php?id=93844\& redirect=1

Cendales, L. y Torres, A. (s.f.). La sistematización como experiencia investigativa y formativa. Disponible en: http://www.cepalforja.org/sistem/documentos/lola_ cendales-alfonso_torres-la_sistematizacion_como_experiencia_investigativa_y_ formativa.pdf

Cendales, L. (2002). Taller 1: Bases teóricas de la sistematización de proyectos económicos. Memorias, Fundación ALBOAN en colaboración con el Instituto de Derechos Humanos Pedro Arrupe y Hegoa. Disponible en: http://62.99.71.146/alboan/ sistematizacion/MemoriaTaller1.pdf

Ghiso, A. (1998). De la práctica singular al diálogo con lo plural. Aproximaciones a otros tránsitos y sentidos de la sistematización en épocas de globalización. Funlam. Disponible en: http://aprendeenlinea.udea.edu.co/lms $/ \mathrm{moodle} / \mathrm{mod} / \mathrm{resource} / \mathrm{view}$. php?id=93899\& redirect $=1$

Jara, O. (2011). La sistematización de experiencias: aspectos teóricos y metodológicos. Entrevista a Oscar Jara. Decisio. Disponible en: http://tumbi.crefal.edu.mx/decisio/ images/pdf/decisio_28/decisio28_testimonios1.pdf

Jara, O. (2012). Sistematización de experiencias, investigación y evaluación: aproximaciones desde tres ángulos. Revista Internacional sobre Investigación en Educación Global y para el Desarrollo. Disponible en: http://educacionglobalresearch. net/wp-content/uploads/02A-Jara-Castellano.pdf

Kuhn, T. (1970). La estructura de las revoluciones cientificas. México: FCE.

Zúñiga, R., Mejía, M., Fernández, B. y Duarte, L. (2015). La sistematización de experiencias en América Latina y el Caribe. Aportes a la práctica docente. Programa Latinoamericano de Apoyo a la Sistematización - Consejo de Educación Popular de América Latina y el Caribe (PLAS-Ceaal). Docencia. №. 55. Disponible en: http://www.revistadocencia.cl/ new/wp-content/pdf/20150514104514.pdf

Mejía, M.R. (s.f.). La sistematización como proceso investigativo o la búsqueda de la episteme de las prácticas. Planeta Paz. Expedición Pedagógica Nacional. Programa Ondas de Colciencias.. Disponible en: http://www.cepalforja.org/sistem/sistem_old/ sistematizacion_como_proceso_investigativo.pdf

Popper, K. (1986). La lógica de la investigación científica. Madrid: Tecnos.

Sánchez, C. (s.f.). La evaluación un asunto político. Seminario Virtual. Caja de Herramientas. Disponible en: http://viva.org.co/cajavirtual/svc0119/index\%20-\%20 pagina\%209.html 
Torres, A. (2011). "La sistematización de experiencias presupuestos epistemológicos y procesos metodológicos". En Páramo, P. La investigación en ciencias sociales. Estrategias de investigación. Bogotá: Universidad Piloto de Colombia.

Vasilachis, I. (2006). Estrategias de investigación cualitativa. Barcelona: Gedisa. 